\title{
ICU management of complicated sepsis secondary to necrotizing fasciitis
}

\author{
Mathias Fricot (Meds 2016) \\ Faculty Reviewer: Dr Lois Champion, MD, FRCPC (Department of Anesthesia) \\ Observed Physician: Dr Philip George, MB, MRCP(UK), FRCPC (Staff Intensivist TBRHSC)
}

\begin{abstract}
In this report we present a case of sepsis secondary to necrotizing fasciitis and its complications.
\end{abstract}

\section{CASE PRESENTATION}

A 37-year-old woman from a Northern Ontario community presented to the nursing station with a 3-day history of gradual-onset swelling and pain in the left arm. Upon arrival the patient was alert, oriented, and had no respiratory, abdominal, or cardiovascular symptoms. She had high-grade fever, chills, and night sweats. The arm was tender and swollen with visible hemorrhagic areas. She is a long-standing IV drug abuser and admitted to missing the vein when she injected drugs 5 days ago. Since then, the patient had noticed reddening of the right arm and complained of flu-like symptoms.

Relevant past medical history included intravenous (IV) drug use, hepatitis C positive status, and narcotic abuse. She had a previous MRSA positive nasal swab during a hospital admission and has a documented penicillin allergy (rash). The patient is a tobacco smoker with a 10-pack-year history.

A provisional diagnosis of severe cellulitis in the left arm was made. The patient was admitted to the local hospital and started on IV ceftriaxone, IV normal saline, ibuprofen, and acetaminophen. Blood cultures and complete blood count (CBC) were ordered. Over the course of the next 3 days a high-grade fever persisted and swelling of the arm worsened in spite of parenteral antibiotics. She developed hypotension and was noted to have worsening renal function on blood tests indicating acute kidney injury. Intravenous fluid resuscitation was commenced, vancomycin and clindamycin were added to the antibiotic regimen, and the patient was transferred to Thunder Bay Regional Health Sciences Centre (TBRHSC) for further management.

Upon arrival at TBRHSC the patient was hemodynamically stable (blood pressure 108/59[75], heart rate [HR] 80+, oxygen saturation $95 \%$, respiratory rate [RR] normal, temperature $35.4^{\circ} \mathrm{C}$ ) after $6 \mathrm{~L}$ of crystalloid resuscitation. A provisional diagnosis of necrotizing fasciitis (NF) was made and plastic surgery was consulted. The patient was taken to the operating room (OR) for emergency surgery. Postoperatively she was extubated and admitted to the intensive care unit (ICU) for ongoing care. A broad-spectrum antibiotic regimen using ceftriaxone, vancomycin, clindamycin, and metronidazole was initiated postoperatively in conjunction with morphine for pain control.
At admission to the ICU the patient was hypotensive, tachypneic (respiratory rate $20 / \mathrm{min}$ ), and intermittently confused with poor urine output. Vital signs were blood pressure 112/72, HR 72, and oxygen saturation $94 \%$ on $4 \mathrm{~L}$ nasal prongs. Lactate levels were elevated at $5 \mathrm{mmol} / \mathrm{L}$. Arterial blood gases (ABG) showed uncompensated metabolic acidosis and hypoxemia. Chest x-ray showed bilateral interstitial shadowing with interspersed areas of focal consolidation. Blood cultures from the previous hospital were positive for gram-positive cocci. A diagnosis of septic shock secondary to NF and complicated by acute respiratory distress syndrome (ARDS) was made.

In the ICU, IV crystalloids and broad-spectrum antibiotics (ceftriaxone, clindamycin, vancomycin) were continued, a central line inserted and vasopressor therapy with norepinephrine initiated. Within a few hours the patient required intubation and mechanical ventilation for increasing oxygen requirements and work of breathing according to the ARDS Network protocol for protective lung ventilation. ICU management also included correction of electrolyte imbalances and close monitoring of fluid status with central venous pressure and central venous oxygen status measurements.

Table 1: Signs/Symptoms Associated With Necrotizing Fasciitis at the Time of Admission ${ }^{a}$

\begin{tabular}{|c|c|c|c|}
\hline FINDING & $\begin{array}{r}\% \text { OF PATIENTS } \\
(n=89)\end{array}$ & $\begin{array}{r}\% \text { OF PATIENTS } \\
(n=192)\end{array}$ & $\begin{array}{r}\% \text { OF PATIENTS } \\
(n=122)\end{array}$ \\
\hline Erythema & 100 & 66 & 95 \\
\hline $\begin{array}{l}\text { Pain or tenderness } \\
\text { beyond margins of } \\
\text { erythema }\end{array}$ & 98 & 73 & 95 \\
\hline Swelling & 92 & 75 & 86 \\
\hline $\begin{array}{l}\text { Crepitus or skin } \\
\text { necrosis }\end{array}$ & 13 & 31 & 0 \\
\hline Induration & 12 & 45 & \\
\hline Bullae & 45 & 23 & 41 \\
\hline Fluctuance & 11 & & \\
\hline Fever & 53 & 32 & \\
\hline Hypotension & 18 & 11 & \\
\hline
\end{tabular}




\section{FEATURE ARTICLE}

Table 2: Diagnostic Criteria for Sepsis. ${ }^{a}$

Diagnosis requires an infection ${ }^{\mathrm{b}}$ (documented or suspected), and some of the following: ${ }^{\mathrm{c}}$

VARIABLE

1. General Variables

\section{CRITERIA}

- Fever (core temperature $>38.3^{\circ} \mathrm{C}$ )

- Hypothermia (core temperature $<36^{\circ} \mathrm{C}$ )

- Heart rate $>90 \mathrm{~min}^{-1}$ or >2 SD above the normal value for age

- Tachypnea

- Altered mental status

- Significant edema or positive fluid balance (>20 mL/kg over $24 \mathrm{~h}$ )

- Hyperglycemia (plasma glucose $>120 \mathrm{mg} / \mathrm{dL}$ or $7.7 \mathrm{mmol} / \mathrm{L}$ ) in the absence of diabetes

\begin{tabular}{|c|c|}
\hline 2. Inflammatory Variables & $\begin{array}{l}\text { - Leukocytosis (WBC count }>12000 \mu \mathrm{L}^{-1} \text { ) } \\
\text { - Leukopenia (WBC count }<4000 \mu \mathrm{L}^{-1} \text { ) } \\
\text { - Normal WBC count with }>10 \% \text { immature forms } \\
\text { - Elevated plasma C-reactive protein ( }>2 \text { SD above the normal value) } \\
\text { - Elevated plasma procalcitonin ( }>2 \mathrm{SD} \text { above the normal value) }\end{array}$ \\
\hline 3. Hemodynamic Variables & $\begin{array}{l}\text { - Arterial hypotension }{ }^{\mathrm{c}}(\mathrm{SBP}<90 \mathrm{mmHg}, \mathrm{MAP}<70 \text {, or decrease in } \mathrm{SBP}>40 \mathrm{mmHg} \text { in adults or }<2 \text { SD } \\
\text { below normal for age) } \\
\text { - Elevated mixed venous } \mathrm{SaO}_{2}(>70 \%) \mathrm{c} \\
\text { - Elevated cardiac index }\left(>3.5 \mathrm{~L} \cdot \mathrm{min}^{-1} \cdot \mathrm{M}^{-23}\right)^{\mathrm{c}}\end{array}$ \\
\hline 4. Organ Dysfunction Variables & $\begin{array}{l}\text { - Arterial hypoxemia }\left(\mathrm{PaO}_{2} / \mathrm{FiO}_{2}<300 \text { ) }\right. \\
\text { - Acute oliguria (urine output }<0.5 \mathrm{~mL} \cdot \mathrm{kg}^{-1} \cdot \mathrm{h}^{-1} \text { or } 45 \mathrm{ml} / \mathrm{h} \text { for at least } 2 \mathrm{~h} \text { ) } \\
\text { - Creatinine increase }>0.5 \mathrm{mg} / \mathrm{dL} \\
\text { - Coagulation abnormalities }(\mathrm{INR}>1.5 \text { or aPPT }>60 \mathrm{~s} \text { ) } \\
\text { - Ileus (absent bowel sounds) } \\
\text { - Thrombocytopenia (platelet count }<100,000 \mathrm{\mu L}^{-1} \text { ) } \\
\text { - Hyperbilirubinemia (plasma total bilirubin }>4 \mathrm{mg} / \mathrm{dL} \text { or } 70 \mathrm{mmol} / \mathrm{L} \text { ) }\end{array}$ \\
\hline
\end{tabular}

5. Tissue Perfursion Variables

- Hyperlactatemia (>1 mmol/L)

- Decreased capillary refill or mottling

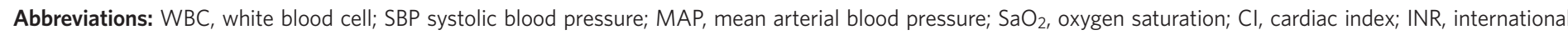

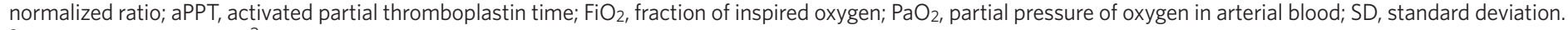
adapted from Levy et al. ${ }^{3}$

${ }^{b}$ Infection defined as a pathologic process induced by a microorganism.

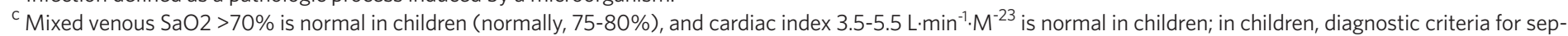

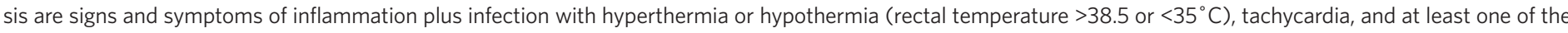
following indications of altered organ function: altered mental status, hypoxemia, increased serum lactate level, or bounding pulses.

\section{DISCUSSION}

Necrotizing fasciitis (NF) is a rapidly progressing infection along fascial planes with secondary infection and necrosis of deep subcutaneous tissues. This rare condition has a mortality rate of up to $35 \%$ with the time to operative intervention being the primary determinant of mortality. ${ }^{1}$ The diagnosis is a clinical one requiring a high index of suspicion (Table 1); treatment of NF is based on the removal of necrotic tissue, drainage, antibiotic therapy, and management of sepsis-related complications. ${ }^{2}$ Antibiotic regimens usually include clindamycin for its anti-toxin effect. ${ }^{2}$ Ceftriaxone is adequate for treating cellulitis but it is not sufficient antibiotic coverage for NF. When a necrotizing soft tissue infection is suspected it is important to have early broad-spectrum antibiotics coverage until cultures become available. The early management in this case highlights the importance of clinical suspicion; if a deeper infection was suspected earlier then perhaps some of the complications successfully managed in the ICU could have been avoided. In the OR, our patient underwent debridement and excision of dead fascia and muscle as source control as well as extensive fasciotomy of the left forearm to prevent compartment syndrome.

Classification of NF is based on microbiology. Type I NF is a mixed infection of aerobic and anaerobic bacteria occurring in patients with several other comorbidities. Type II NF is caused by group A streptococcus alone or in combination with another species, commonly Staphylococcus aureus. ${ }^{1}$ This is less common than type I NF and typically occurs in otherwise healthy patients with a predisposing skin injury. ${ }^{1}$ The complications of NF include sepsis related to its systemic toxicity, compartment syndrome from small vessel thrombosis, limb loss, and death.

Sepsis is defined as the combination of systemic inflammatory response syndrome (SIRS) and a source of infection. SIRS is clinical syndrome characterized by two or more of the following; tachycardia ( $\mathrm{HR}>90 / \mathrm{min}$ ), tachypnea ( $\mathrm{RR}>20 / \mathrm{min}$ ), altered leukocytes (WBC $<4 \times 10^{9}$ or $\left.>12 \times 10^{9}\right)$, or altered body temperature $\left(<36^{\circ} \mathrm{C}\right.$ or $\left.\left.>38^{\circ} \mathrm{C}\right)\right)^{3,4}$ International guidelines provide a list of warning signs for recognizing developing sepsis (Table 2). ${ }^{4}$

Management of sepsis is divided in two parts: initial early goal-directed therapy (EGDT) to be accomplished within the first 6 hours and maintenance therapy in the ICU. ${ }^{4}$ This strategy has been 


\title{
FEATURE ARTICLE
}

\author{
Table 3: Berlin Definition of Acute Respiratory Distress Syndrome ${ }^{a}$

\begin{tabular}{lll}
\hline 1. Timing & - Within one week of a known clinical insult or new/worsening respiratory symptoms \\
\hline 2. Chest Imaging ${ }^{b}$ & Bilateral opacities - not fully explained by effusions, lobar/lung collapse, or nodules
\end{tabular} \\ 3. Origin of Edema \\ 4. Oxygenation ${ }^{\mathrm{c}}$ \\ - Respiratory failure not fully explained by cardiac failure or fluid overload; need objective assessment \\ (eg echocardiography) to exclude hydrostatic edema if no risk factor present

\begin{abstract}
PEEP, positive end-expiratory pressure.
adapted from ARDS Definition Task Force. ${ }^{17}$

${ }^{\mathrm{b}}$ Chest radiograph or computed tomography scan.

${ }^{c}$ If altitude $>1000 \mathrm{~m}$, correction factor should be made as follows: $\mathrm{PaO}_{2} / \mathrm{FiO}_{2}$. (barometric pressute/760).

$\mathrm{d}$ This may be delivered noninvasively in the mild acute respiratory distress syndrome group.
\end{abstract} \\ Abbreviations: CPAP, continuous positive airway pressure; $\mathrm{FiO}_{2}$, fraction of inspired oxygen; $\mathrm{PaO}_{2}$, partial pressure of oxygen in arterial blood; \\ - Mild: $200<\mathrm{PaO}_{2} / \mathrm{FiO}_{2} \leq 300$, with PEEP or CPAP $\geq 5 \mathrm{~cm} \mathrm{H} \mathrm{H}_{2} \mathrm{Od}$ \\ - Moderate: $100<\mathrm{PaO}_{2} / \mathrm{FiO}_{2} \leq 200$, with PEEP or CPAP $\geq 5 \mathrm{~cm} \mathrm{H} \mathrm{H}_{2} \mathrm{O}$ \\ - Severe: $\mathrm{PaO}_{2} / \mathrm{FiO}_{2} \leq 100$, with PEEP or CPAP $\geq 5 \mathrm{~cm} \mathrm{H} \mathrm{H}_{2} \mathrm{O}$
}

shown to reduce mortality. ${ }^{4,5}$

Early management focuses on prompt antimicrobial therapy, treating the source of infection, and cardiorespiratory resuscitation to prevent oxygen deprivation in peripheral tissues. ${ }^{4}$ EGDT central venous pressure targets are maintained through IV fluids, mean arterial pressure targets through vasopressors, and continued oxygen delivery targets through transfusion of red cells, inotropic agents, and mechanical ventilation. ${ }^{4}$

ICU sepsis management focuses on continuing to maintain the established goals of EGDT. ${ }^{4}$ This includes careful monitoring of organ function with necessary supportive therapies and the de-escalation of treatment whenever possible. The Surviving Sepsis Campaign (2012) provides standard guidelines for timely initiation of EGDT and ongoing management. ${ }^{4}$

Severe sepsis is sepsis complicated by acute organ dysfunction and has a mortality rate of approximately $30 \% .{ }^{6}$ The variability of organ systems affected contributes to the highly variable clinical manifestations of sepsis, including central nervous system dysfunction and acute kidney injury (AKI). During early management of sepsis, the lactate present in serum is assumed to be the byproduct of anaerobic metabolism and can be used to gauge organ hypoperfusion. ${ }^{5}$ Organ dysfunction commonly affects the cardiovascular and respiratory systems, which classically manifests as ARDS. ${ }^{7}$

ARDS is a diffuse acute inflammatory lung injury with a recognizable injury pattern involving discontinuous endothelial injury, non-cardiogenic pulmonary edema, and atelectasis throughout both lungs. ${ }^{8}$ Together, these lead to an inhibition of gas exchange and contribute to a clinical picture marked by hypoxemia and bilateral opacities on chest radiography. Pathologically, ARDS correlates with diffuse alveolar damage and has an approximate short-term mortality of $45 \% .{ }^{8}$ Diagnosis of ARDS in our patient was consistent with the Berlin Definition (Table 3$)^{8}$

Management of ARDS focuses on supportive therapy and the treatment of hypoxia using mechanical ventilation. Supportive care involves sedation, hemodynamic monitoring, nutritional support, glucose control, and prevention of complications. ${ }^{8,9}$ Our patient was sedated with continuous propofol infusion and bolus doses of midazolam, given the history of ongoing alcohol abuse. Morphine infusion was continued for ongoing pain control. Standard ventilator management involves a lung-protective ventilation strategy and in our patient followed the ARDS Network protocol. ${ }^{10}$ As ARDS affects the lung heterogeneously, some areas of the lung remain aerated and relatively normal and are sometimes referred to as "baby lungs." The ARDS Network protocol incorporates a lower tidal volume strategy ( $6 \mathrm{cc} / \mathrm{kg}$ ideal body weight) to avoid overdistension of the functioning portion of the lungs and ventilator-induced lung injury. ${ }^{8}$

Patients being treated for ARDS are at a high risk for ventilator-associated complications, notably barotrauma from the positive pressure, volutrauma from excessive tidal volumes, atelectrauma from repeated opening and closing of the alveoli, biotrauma from the release of inflammatory mediators into the circulation, and ventilator-associated pneumonia. ${ }^{8}$ The mortality form ARDS is typically due to associated multi organ dysfunction syndrome (MODS). ${ }^{9}$

The presence of ARDS in our patient presented another challenge for successful management of the patient's sepsis. The AKI was thought to be prerenal in origin, secondary to hypoperfusion during sepsis. Treatment of AKI was source control and IV fluids to maintain enough perfusion pressure. Maintenance of adequate volume status was key; keeping the patient dry enough to prevent pulmonary edema while maintaining adequate fluids to keep the kidneys perfused was a challenge.

\section{CONCLUSION}

The patient's clinical condition stabilized and improvements were seen in urine output, serum creatinine and blood urea nitrogen (BUN). Over the next 2 days vasopressor support was titrated off and the patient returned to the OR for repeat debridement and vacuum-assisted closure dressing changes. Prior to the OR the patient was thrombocytopenic and required 2 units of platelet transfusion to reduce risk of bleeding. The thrombocytopenia was attributed to complicated sepsis. ${ }^{7}$ Blood and wound cultures subsequently came back positive for group A streptococcus and methicillin-resistant 


\section{FEATURE ARTICLE}

Staphylococcus aureus (MRSA). ${ }^{11,12,13}$ The patient's antibiotic regimen was changed to cefazolin, vancomycin, and clindamycin as per the sensitivity results.

The patient was eventually extubated 4 days after ICU admission. Postextubation she continued to improve with normalization of renal function. She required two more OR visits for subsequent debridements. Fortunately, the limb could be salvaged and the patient was eventually discharged to the floor. Antibiotics were continued on a 4 -week course. The fact that this patient survived and did not require an amputation highlights the need for timely and effective surgical intervention in necrotizing fasciitis and the importance of supportive ICU care in recognizing and managing its complications.

\section{REFERENCES}

1. Sarani B, Strong M, Pascual J, Schwab C. Necrotizing fasciitis: current concepts and review of the literature. J Am Coll Surg. 2009;208:279-88.

2. Anaya DA, Dellinger EP. Necrotizing soft-tissue infection: diagnosis and management. Clin Infect Dis. 2007;44:705.

3. Levy MM, Fink MP, Marshall JC, et al. 2001 SCCM/ESICM/ ACCP/ATS/SIS international sepsis definitions conference. Crit Care Med. 2003;31:1250-56

4. Dellinger RP, Levy MM, Rhodes A, et al. Surviving sepsis campaign: international guidelines for management of severe sepsis and septic shock. 2012. Crit Care Med. 2013;41:580-637.

5. Rivers E, Nguyen B, Havstad S, et al. Early goal-directed therapy in the treatment of severe sepsis and septic shock. N Engl J Med. 2001;345:1368-77.

6. Kumar G, Kumar N, Taneja A, et al. Nationwide trends of severe sepsis in the 21st century (2000-2007). Chest. 2011;140:1223-31.

7. Angus DC, Van der Poll R. Severe sepsis and septic shock. N Engl J Med. 2013;369:840-51.
8. Silverside JA, Ferguson ND. Clinical review: Acute respiratory distress syndrome - clinical ventilator management and adjunct therapy. Crit Care. 2013;17:225.

9. Leaver SK, Evans TW. Clinical review: Acute respiratory distress syndrome. BMJ. 2007;336:389-94.

10. The Acute Respiratory Distress Syndrome Network. Ventilation with lower tidal volumes as compared with traditional tidal volumes for acute lung injury and the acute respiratory distress syndrome. N Engl J Med. 2000;342:1301-08.

11. Liu C, Bayer A, Cosgrove SE, et al. Clinical practice guidelines by the Infectious Diseases Society of America for the treatment of methicillin-resistant Staphylococcus aureus infections in adults and children: executive summary. Clin Infect Dis 2011;52:285-92.

12. Rybak M, Lomaestro B, Rotschafer JC, et al Therapeutic monitoring of vancomycin in adult patients: a consensus review of the American Society of Health-System Pharmacists, the Infectious Diseases Society of America, and the Society of Infectious Diseases Pharmacists. Am J Health Syst Pharm 2009;66:82-98.

13. Stevens DL, Bisno AL, Chambers HF, et al Practice guidelines for the diagnosis and management of skin and soft-tissue infections. Clin Infect Dis 2005;41:1373-406.

14. Wong CH, Haw-Chong C, Shanker P, et al. Necrotizing fasciitis: clinical presentation, microbiology, and determinants of mortality. J Bone Joint Surg Am 2003;85:1454-60.

15. Elliot DC, Kufera JA, Myers RA. Necrotizing soft tissue infections. Risk factors for mortality and strategies for management. Ann Surg 1996;224:672-83.

16. Wang YS, Wong CH, Tay YK. Staging of necrotizing fasciitis based on the evolving cutaneous features. Int J Dermatol 2007;46:1036-41.

17. ARDS Definition Task Force, Ranieri VM, Rubenfeld GD, Thompson BT, Ferguson ND, Caldwell E, Fan E, Camporota L, Slutsky AS. Acute respiratory distress syndrome: the Berlin Definition. JAMA 2012;307:2526-33. 